\title{
PRACTICAL IMPLEMENTION OF GAOPF ON INDIAN 220KV TRANSMISSION SYSTEM
}

\author{
C.M. Wankhade ${ }^{1}$ and A.P. vaidya ${ }^{2}$ \\ ${ }^{1}$ Department of Electrical Engineering, L.T. College of Engineering, Koparkhairane, \\ Navi Mumbai \\ ${ }^{2}$ Department of Electrical Engineering, Walchand College of Engineering, Sangli.
}

\begin{abstract}
This paper presents the practical implementation of developed genetic algorithm based optimal power flow algorithms. These algorithms are tested on IEEE3O bus system and the results were presented in the paper [8]. The same algorithms now tested on 220KV Washi zone Indian power transmission system. The GAOPF with fixed penalty and Fuzzy based variable penalty tested on 220KV transmission system consists of 52 bus and 88lines. The fuel costs, computational time and the system condition were studied and the results are presented in this paper. Also the available load transfer capability of the $220 \mathrm{KV}$ system for congestion management is also presented
\end{abstract}

\section{KEYWORDS}

Genetic Algorithm, Optimal Power Flow, Network condition, Generation Cost, Fuzzy Penalty, Transfer Capability, Congestion Management.

\section{INTRODUCTION}

Optimization means the minimizing the generator -operating cost within the given constraints of a transmission system in which the generators are operating [1].The basic principle of mechanics of nature and natural genetics are used in the GA. To get the optimal solution, the mixing of evaluation with randomized, structured exchanges of information is helpful. The use of genetic algorithm load flow with the gradient information by the steepest decent method enhances the result of problem in GAOPF [2]. The transformer taps shunt devices, voltages and generator active power outputs are the control variables used in a GA. In GA, the Fitness Function (FF) based on the term quadratic penalty use the branch flow, reactive generation and the voltage magnitude constraints. The optimal power dispatch problem of a multimode auction market is solved by GA [3]. The nodal real and reactive power injections that clear the market are selected as the problem control variables .A best Result using only simple genetic operations such as proportionate reproduction, simple mutation, and one-point crossover in binary codes are used in a simple genetic algorithm.[4].The selection operator works with the fitness value, any selection operator used with binary coded GAs can also be used in real parameter GAs [5][8], where the Hybrid genetic algorithm (GA) base approach, continuous variables are designed using real-coded GA and discrete variables are processed as binary coded GA. Whereas Real Coded GA has real parameters can be used intact and crossover and mutation operators are applied directly to real parameter values.

Now a day the Congestion management is done with the rescheduling and / or load shedding schemes which avoids the overloading in a transmission lines. For the effective congestion 
management in a power system with the existing transmission and operational constraints are done by using OPF techniques [9]-[10].

\section{OPTIMAL POWER FLOW PROBLEM STATEMENT}

In a optimal power flow certain control variables are adjusted to minimize an objective function by satisfying the physical and operating limits on state and control variable to get the power flow solution.

The minimum fuel cost problem is stated as

Minimize $F=\sum_{i}^{N_{g}}\left(a_{i} P_{g i}^{2}+b_{i} P_{g i}+c\right) \quad \$ / \mathrm{hr}$

\section{BRIEF INTRODUCTION TO GENETIC ALGORITHM}

Genetic Algorithm is used for global function/control optimization based on the theory of survival of the fittest. The three basic operator's are-Reproduction, Cross-over and Mutation .Firstly the GA generate the initial population. Each member (individual) of the population is associated with binary string of suitable length L. A solution of the problem is in the string. For intermediate population a sampling of initial population are used. Crossover explores new regions in the search space. Crossover is responsible for the structure recombination and the Mutation is used to avoid premature convergence of population and to fine tune the solution. A maximum fitness value in the searching process provides the minimum fitness function. For a larger fitness value a chromosome of lower cost function are used. The maximization of fitness in Simulated Roulette wheel is the objective of OPF.

Fitness Function $\mathrm{ff}=\frac{1}{1+\mathrm{f}^{2}}$

Where,

$$
f=f_{C}+P_{1}\left(\sum h^{2}(x, u)\right)+P_{2}\left(\sum g^{2}(x, u)\right)
$$

\section{PROPOSED ALGORITHMS FOR SOLUTION OF OPF}

Equation (1) will provide the solution to optimal power flow by adjusting the control variable $u$ in a specific search direction satisfying the equality and inequality constraints. The selection of control and state variables and the techniques define the convergence and time for convergence. The solution time depends on the size of the system. OPF with voltages as control vectors posses a slow convergence rate for GA, as GA is random population based search algorithm. In this case, the GA utilizes large generation and without guaranteed convergence. Considering, the disadvantages of it, this paper suggests, 3 algorithms which speeds the solution and accuracy.

Let,

$\mathrm{U}=\{\mathrm{V}, \delta, \mathrm{P}, \mathrm{Q}\}$

Where, 
$\mathrm{V}$ - the set voltage magnitudes of buses in a power system

$\delta$ - the set angles of buses in a power system

$\mathrm{P}$ - the set of active power

$\mathrm{Q}$ - the set of reactive power

Let, $\mathrm{B}$ is set defined as,

$\mathrm{B}=\{\mathrm{G}, \mathrm{L}, \mathrm{C}, \mathrm{TL}, \mathrm{T}\}$

Where,

$\mathrm{G}$ - set of generating buses $\mathrm{G}$

$\mathrm{L}$ - set of load buses and

C - set of controlled buses

$\mathrm{TL}$ - the set of transmission lines

$\mathrm{T}$ - set of transformers

Let, $M G \in G$, is maximum generation capability bus which will supply the losses in power system.

\subsection{GA Based Approach}

\subsection{1 - AlgORITHM (A):}

Many optimization applications use GA as global optimization techniques. The conventional algorithm of OPF suffers from disadvantage of getting trapped into local optimum; hence the GA is used to obtain the solution of OPF. Increase in the number of OPF variables slows the convergence rate for GA, that's why the selection of control variable is critical issue in GA application of OPF. Sets of control variables were tested extensively to find the effect of control variable on the convergence of simple genetic algorithm.

The wide spread control variables used are chosen to find the optimum solution as

$$
\begin{aligned}
& u=\left[\begin{array}{ll}
V_{G} & P_{G}
\end{array}\right]^{T} \\
& X=\left[\begin{array}{lllll}
P_{T L} & Q_{T L} & V_{L} & \delta & Q_{G}
\end{array}\right]^{T}
\end{aligned}
$$

The penalty function is used to improve the convergence criterion of Simple GA in this algorithm. The fitness function which is to be minimized is given by

$$
\mathrm{F}=\mathrm{f}_{\mathrm{c}}+\sum_{\mathrm{i}=1}^{\mathrm{p}} \mathrm{P}_{\mathrm{eq}}\left[\mathrm{g}_{\mathrm{i}}(\mathrm{x}, \mathrm{u})\right]^{2}+\sum_{\mathrm{j}=1}^{\mathrm{m}} \lambda_{\text {ineq }}\left[\mathrm{h}_{\mathrm{j}}(\mathrm{x}, \mathrm{u})\right]^{2}
$$

OR

$$
\begin{aligned}
& F=f_{c}+P_{e q} \sum_{i=1}^{p}\left[G_{i}(x, u)\right]^{2}+P_{\text {ineq }} \sum_{j=1}^{m}\left[H_{j}(x, u)\right]^{2} \text { Where } \\
& {\left[G_{i}(x, u)\right]^{2}=g_{i}{ }_{i}^{2}(x, u)}
\end{aligned}
$$




$$
\left[\mathrm{H}_{\mathrm{j}}(\mathrm{x}, \mathrm{u})\right]^{2}=\left(\max \left[0, \mathrm{~h}_{\mathrm{j}}(\mathrm{x}, \mathrm{u})\right]\right)^{2}
$$

Where $P_{e q}$ and $P_{\text {ineq }}$ are the Penalty terms for the equality and inequality constraints. With the assumed control variables, the state variables $\mathrm{X}$ of system are obtained by using fast decoupled load flow solution, by iteratively solving the equation for load flow solution.

$$
\left[\begin{array}{c}
\Delta \mathrm{P} \\
\Delta \mathrm{Q}
\end{array}\right]=\left[\begin{array}{cc}
\mathrm{H} & 0 \\
0 & \mathrm{~L}
\end{array}\right]\left[\begin{array}{c}
\Delta \delta \\
\Delta \mathrm{V}
\end{array}\right]
$$

Load flow solution is used to obtain fitness function given in equation number (8) OR (9). To change the search direction the cross-over and mutation are carried out on the population.

Whenever the constraint is violated, the penalty $(+)$ being added which is proportional to square of the amount of violation while minimizing the objective fitness function.

\subsubsection{Algorithm (B):}

Where bus switching is allowed during the fast decoupled load flow calculations.

\subsubsection{Algorithm $(C)$ :}

More practical set of control variables are chosen in this algorithm (C).

$$
\begin{aligned}
& \mathrm{u}=\left[\begin{array}{llll}
V_{M G} & P_{G} & Q_{G} & Q_{C}
\end{array}\right]^{T} \\
& x=\left[\begin{array}{llll}
P_{T L} & Q_{T L} & V_{G \notin M G} & V_{C} \\
V_{L} & \delta
\end{array}\right]^{T}
\end{aligned}
$$

Conditions of Algorithm (A) and of Algorithm (B) are used in Algorithm (C). The fuzzy penalty (FP) is also applied to all algorithms such as PV-FP (without switching), PV-SW-FP (with switching) and PVQ- FP.

\section{CASE STUDY}

The effect of widespread control variables on the OPF solution of Indian practical 220KV Washi Zone Transmission System of 52 bus and 88 lines were studied. The suggested algorithms were tested under both normal and contingent conditions. For one transmission line total 88 contingencies are considered during the study of Fig (5.1). 


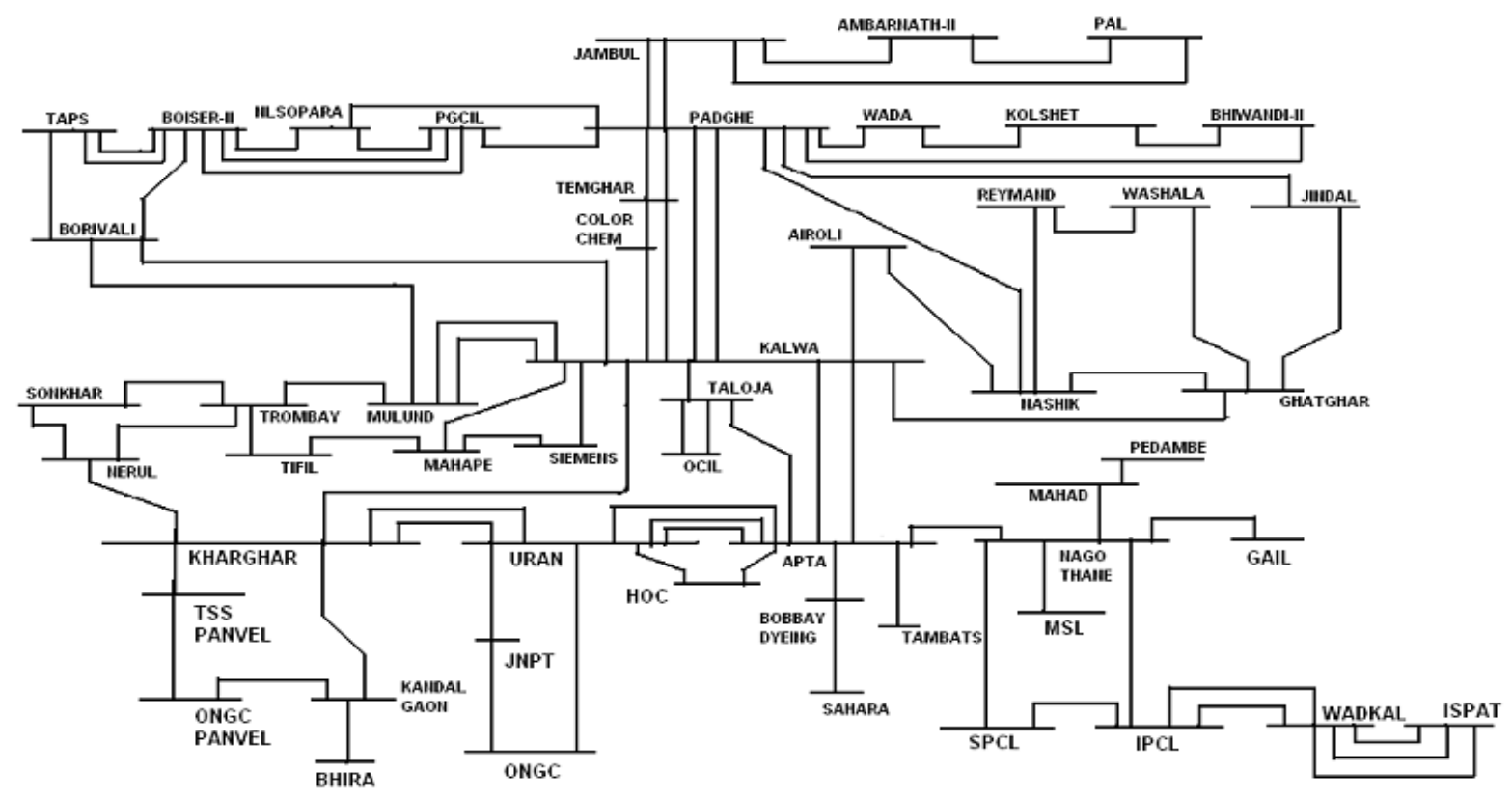

Fig (5.1).Indian Practical Washi Zone 220kV Transmission Network

Similar study like IEEE-30 bus system is also been carried out on the Indian $220 \mathrm{kV}$ WashiZone transmission system of 52 bus and 88 lines.

\section{SGA based OPF using Fixed Penalty}

The proposed algorithms are analyzed for cost of generation, computational time, system condition and transmission line flows of the system.

\subsection{Cost of generation}

Result of the SGA based proposed algorithms with fixed penalty under with and without contingency conditions is given in table (5.1) and (5.2) respectively.

Table (5.1): Fuel cost comparison based on statistical parameters under without contingency condition

\begin{tabular}{|l|l|l|l|l|l|l|}
\hline \multirow{3}{*}{ Parameter } & \multicolumn{4}{|l|}{$\mathrm{CV}=0.1, \mu=0.1$} & \multicolumn{2}{l|}{$\mathrm{CV}=0.7, \mu=0.5$} \\
\cline { 2 - 7 } & $\begin{array}{l}\text { PROAL- } \\
\text { PV }\end{array}$ & $\begin{array}{l}\text { PROAL- } \\
\text { PV-SW }\end{array}$ & $\begin{array}{l}\text { PROAL- } \\
\text { PVQ }\end{array}$ & $\begin{array}{l}\text { PROAL- } \\
\text { PV }\end{array}$ & $\begin{array}{l}\text { PROAL- } \\
\text { PV-SW }\end{array}$ & $\begin{array}{l}\text { PROAL- } \\
\text { PVQ }\end{array}$ \\
\hline Average & 8.90 & 9.46 & 8.96 & 9.67 & 9.39 & 8.95 \\
\hline Minimum & 8.90 & 8.85 & 8.86 & 9.15 & 9.09 & 8.91 \\
\hline Maximum & 8.90 & 9.53 & 9.59 & 10.19 & 9.42 & 8.96 \\
\hline Kurtosis & 1.00 & 8.11 & 7.88 & 1.00 & 8.11 & 3.47 \\
\hline Skewness & -1.00 & -2.66 & 2.59 & -0.003 & -2.66 & -1.22 \\
\hline Std. deviation & 0.00 & 0.215 & 0.22 & 0.54 & 0.105 & 0.016 \\
\hline
\end{tabular}


Table (5.2): Comparison of Fuel Cost based on statistical parameters under contingency condition (line 64)

\begin{tabular}{|l|l|l|l|l|l|l|}
\hline \multirow{2}{*}{ Parameter } & \multicolumn{3}{|l|}{$\mathrm{CV}=0.1, \mu=0.1$} & \multicolumn{2}{l|}{$\mathrm{CV}=0.7, \mu=0.5$} \\
\cline { 2 - 7 } & $\begin{array}{l}\text { PROAL- } \\
\text { PV }\end{array}$ & $\begin{array}{l}\text { PROAL- } \\
\text { PV-SW }\end{array}$ & $\begin{array}{l}\text { PROAL- } \\
\text { PVQ }\end{array}$ & PROAL-PV & $\begin{array}{l}\text { PROAL- } \\
\text { PV-SW }\end{array}$ & PROAL-PVQ \\
\hline Average & 10.83 & 10.65 & 10.67 & 11.63 & 11.00 & 10.67 \\
\hline Minimum & 10.82 & 10.64 & 10.53 & 10.76 & 10.66 & 10.62 \\
\hline Maximum & 10.83 & 10.68 & 11.42 & 12.85 & 14.05 & 10.70 \\
\hline Kurtosis & 1.16 & 8.11 & 7.94 & 1.17 & 8.11 & 1.59 \\
\hline Skewness & 0.40 & 2.66 & 2.61 & 0.38 & 2.66 & -0.67 \\
\hline Std. deviation & 0.001 & 0.011 & 0.26 & 1.05 & 1.07 & 0.039 \\
\hline
\end{tabular}

The spread of convergence values (standard deviation) of fuel cost found to be minimum as the tendency of PROAL-PVQ has to converge towards global optimum than all other algorithms at higher cross-over and mutation probabilities. The algorithm PROAL-PVQ provides better estimate of average value Rs. $8.96 / \mathrm{KWh}$ for $\mathrm{CV}=0.1$ and $\mu=0.1$ and Rs. $8.95 / \mathrm{KWh}$ for $\mathrm{CV}=0.7$ and $\mu=0.5$, from table (5.1).

From table (5.2), it is observed that algorithm PROAL-PVQ provides better estimate of fuel cost in comparisons with other algorithms. The standard deviation of objective function obtained for algorithm PROAL-PVQ in 10 runs is 0.26 and 0.04 under ordered pair $(0.1,0.1)$ and $(0.7,0.5)$ respectively.

Table (5.3) shows the comparative fuel cost values for the various GA and classical approach based algorithms under specific transmission line contingencies. Algorithm LMMS-PQV $\delta$ provides best estimation of fuel cost under no contingency condition, while other provides slightly higher estimates. In transmission line contingency no. 64, all the classical approach algorithms fail to converge. This shows the utility of GA based algorithm in solution of OPF. For this specific condition algorithm PROAL-PVQ provides minimum average fuel cost (Rs. 10.67) amongst the all other algorithm .This shows that the importance of selection of control variables for the solution of OPF.

Table (5.3): Comparative fuel cost for all proposed algorithms $(\mathrm{CV}=0.7, \mu=0.5)$

\begin{tabular}{|l|l|l|l|l|l|l|}
\hline TLC & PROAL-PV & PROAL-PV-SW & PROAL-PVQ & NBOPF & LMMS & $\begin{array}{l}\text { LMMS- } \\
\text { PQV } \delta\end{array}$ \\
\hline 0 & 9.67 & 9.39 & 8.95 & 7.89 & 10.15 & 7.6343 \\
\hline 64 & 11.63 & 11.00 & 10.67 & NC & NC & NC \\
\hline
\end{tabular}

\subsubsection{Computation Time}

The average, minimum and maximum computational time obtained by various GA based algorithms under no contingency and contingency conditions for various probability ordered pairs are given in table (5.4) and table (5.5) respectively. 
Table (5.4): Comparison of computational time based on statistical parameters under no contingency condition

\begin{tabular}{|c|c|c|c|c|c|c|}
\hline \multirow[b]{2}{*}{ Parameter } & \multicolumn{3}{|c|}{$\mathrm{CV}=0.1, \mu=0.1$} & \multicolumn{3}{|c|}{$\mathrm{CV}=0.7, \mu=0.5$} \\
\hline & $\begin{array}{l}\text { PROAL- } \\
\text { PV }\end{array}$ & $\begin{array}{l}\text { PROAL- } \\
\text { PV-SW }\end{array}$ & $\begin{array}{l}\text { PROAL- } \\
\text { PVQ }\end{array}$ & $\begin{array}{l}\text { PROAL- } \\
\text { PV }\end{array}$ & $\begin{array}{l}\text { PROAL- } \\
\text { PV-SW }\end{array}$ & $\begin{array}{l}\text { PROAL- } \\
\text { PVQ }\end{array}$ \\
\hline Average & 81.63 & 94.22 & 69.44 & 77.98 & 97.17 & 59.93 \\
\hline Minimum & 80.20 & 86.01 & 39.57 & 52.78 & 88.12 & 54.01 \\
\hline Maximum & 83.25 & 144.5 & 87.21 & 94.39 & 110.84 & 64.35 \\
\hline
\end{tabular}

Under severe contingency condition, it can be observed from the table (5.5), algorithm PROALPVQ utilizes the minimum time as compared to the other algorithms.

Table (5.5): Computational time comparison based on statistical parameters under contingent condition of line 64

\begin{tabular}{|c|c|c|c|c|c|c|}
\hline \multirow[b]{2}{*}{ Parameter } & \multicolumn{3}{|c|}{$\mathrm{CV}=0.1, \mu=0.1$} & \multicolumn{3}{|c|}{$\mathrm{CV}=0.7, \mu=0.5$} \\
\hline & $\begin{array}{l}\text { PROAL- } \\
\text { PV }\end{array}$ & $\begin{array}{l}\text { PROAL- } \\
\text { PV-SW }\end{array}$ & $\begin{array}{l}\text { PROAL- } \\
\text { PVQ }\end{array}$ & $\begin{array}{l}\text { PROAL- } \\
\text { PV }\end{array}$ & $\begin{array}{l}\text { PROAL- } \\
\text { PV-SW }\end{array}$ & $\begin{array}{l}\text { PROAL- } \\
\text { PVQ }\end{array}$ \\
\hline Average & 154.80 & 108.16 & 59.92 & 80.23 & 89.22 & 65.81 \\
\hline Minimum & 84.17 & 98.14 & 48.125 & 57.98 & 78.42 & 53.125 \\
\hline Maximum & 206.89 & 126.9 & 75.31 & 95.25 & 96.56 & 87.54 \\
\hline
\end{tabular}

\subsubsection{Effect on system conditions}

Analysis of $220 \mathrm{kV}$ network for various system conditions using the SGA based OPF is presented in the next section.

\subsubsection{Bus Voltage Profile}

The bus voltage profile obtained with and without the contingency conditions is given in figure (5.2) and (5.3) respectively.

From figure (5.2), the bus voltages obtained through algorithms PROAL-PV and PROAL-PVSW violates the voltage security constraints of $10 \%$ voltage regulation for the buses 19 to 25 , while the algorithm PROAL-PVQ satisfies the voltage regulation limits. Under contingency condition, PROAL-PVQ selects the higher voltages for systems buses to satisfy the optimal power flow conditions.

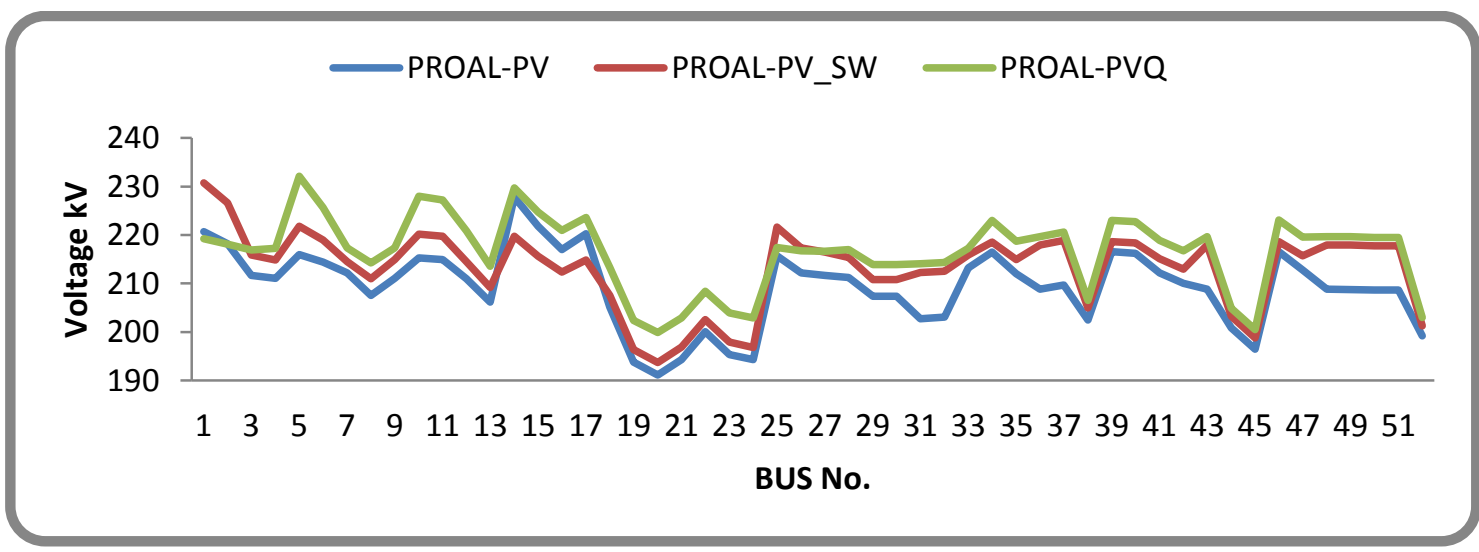

Figure (5.2) chart for voltage profile of buses under no contingency condition 
The figure (5.3) shows the comparative chart for voltage profile under transmission line contingency number 64 , where the conventional approaches fails to provide the solution. It can be observed from the figure that algorithm PROAL-PVQ with fixed penalty selects the higher voltage for buses than the other two proposed algorithms.

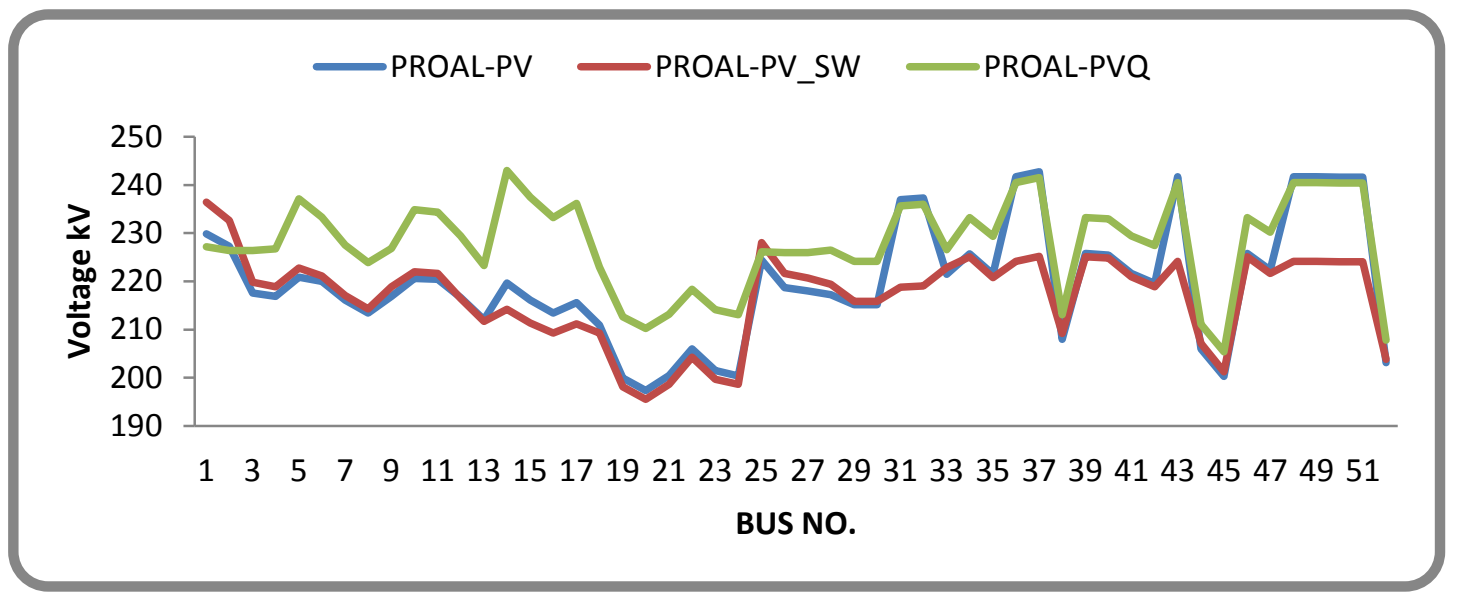

Figure (5.3): Comparison chart for voltage profile of buses under contingency condition of line 64

\subsubsection{Allocation of Real power generation}

It can be observed from table (5.6) that, the LMMS-PQV $\delta$ selects the generations from the few of the generating buses based on their cost of generation. This algorithm provides the best optimal solution in view of percentage loss than other algorithm under no contingency condition. The \% loss calculated at this condition by this algorithm is smallest amongst the all. The graphical representation of the comparative \% generation allocation from each bus is shown in figure (5.4). Comparatively, loss calculated by PROAL-PVQ is $0.91 \%$ which is smallest amongst the PROAL-PV, PROAL-PV-SW or LMMS.

Table (5.6): \% Real Power generation allocation during without contingency condition

\begin{tabular}{|l|l|l|l|l|l|l|}
\hline Bus No. & $\begin{array}{l}\text { PROAL- } \\
\text { PV }\end{array}$ & $\begin{array}{l}\text { PROAL-PV- } \\
\text { SW }\end{array}$ & PROAL-PVQ & NBOPF & LMMS & $\begin{array}{l}\text { LMMS- } \\
\text { PQV } \delta\end{array}$ \\
\hline 1 & 22.00 & 21.74 & 22.49 & 52.97 & 19.94 & 32.35 \\
\hline 4 & 3.41 & 7.28 & 4.88 & 0.00 & 8.41 & 0.00 \\
\hline 5 & 11.33 & 10.48 & 10.19 & 28.88 & 11.53 & 29.00 \\
\hline 14 & 13.32 & 8.58 & 14.67 & 5.33 & 15.66 & 5.33 \\
\hline 18 & 15.59 & 16.46 & 14.48 & 0.00 & 27.36 & 0.00 \\
\hline 33 & 1.17 & 1.86 & 0.41 & 0.00 & 6.63 & 0.00 \\
\hline 34 & 28.35 & 28.38 & 27.95 & 2.00 & 2.00 & 28.39 \\
\hline 36 & 1.00 & 1.45 & 0.89 & 0.00 & 2.75 & 0.00 \\
\hline 52 & 4.99 & 4.98 & 4.95 & 11.33 & 6.71 & 5.00 \\
\hline $\begin{array}{l}\text { \% Total } \\
\text { Gen. }\end{array}$ & 101.16 & 101.21 & 100.91 & 100.52 & 100.99 & 100.07 \\
\hline $\begin{array}{l}\text { \% Total } \\
\text { Load }\end{array}$ & 100.00 & 100.00 & 100.00 & 100.00 & 100.00 & 100.00 \\
\hline \% Loss & 1.16 & 1.21 & 0.91 & 0.52 & 0.99 & 0.07 \\
\hline
\end{tabular}




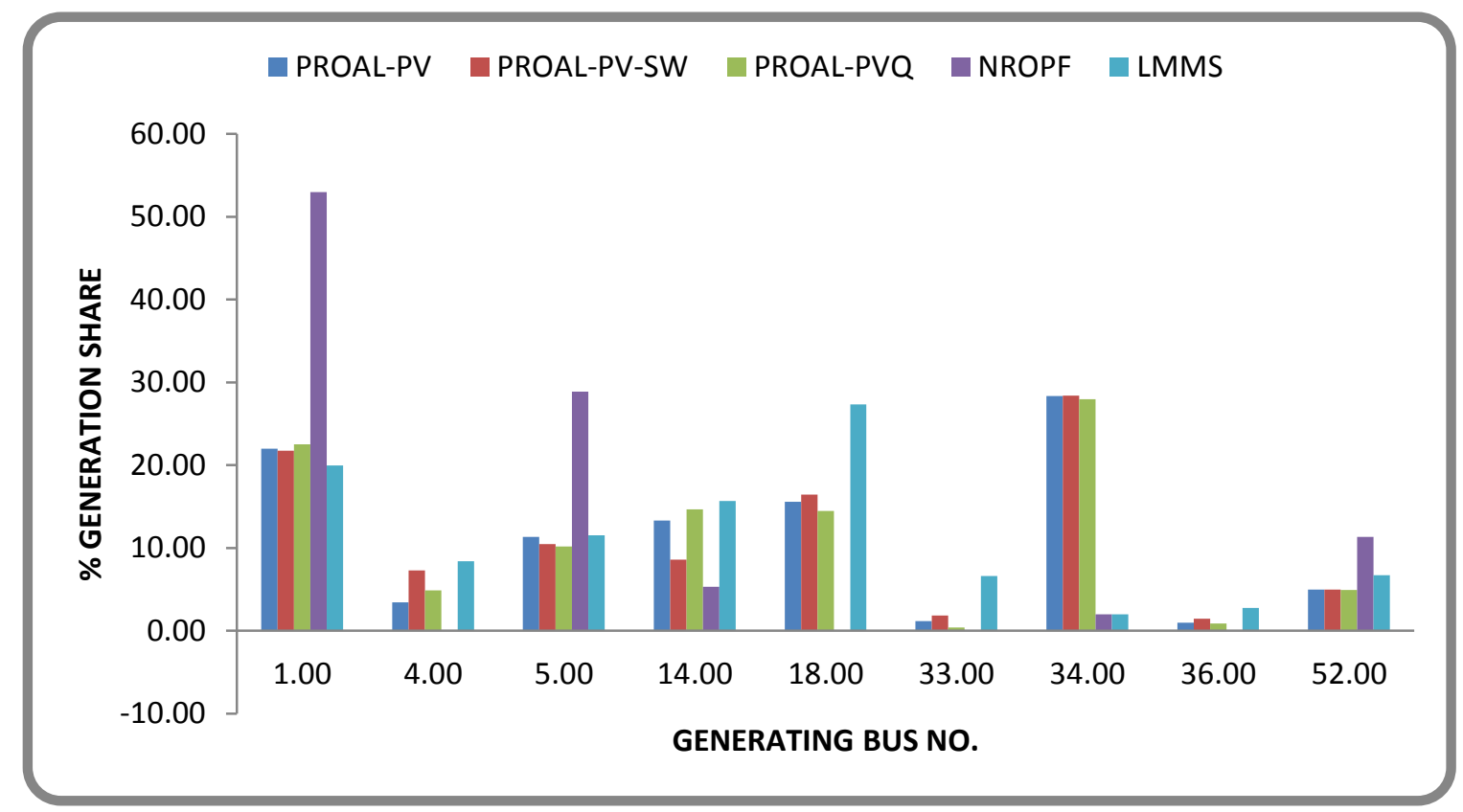

Figure (5.4): \% Real Power generation allocation during without contingency condition

While in contingent conditions, the generation taken by the SGA based proposed algorithms is shown in table (5.7). The same is represented in figure (5.5). It can be seen that, \% loss obtained from PROAL-PVQ is smallest amongst the algorithms.

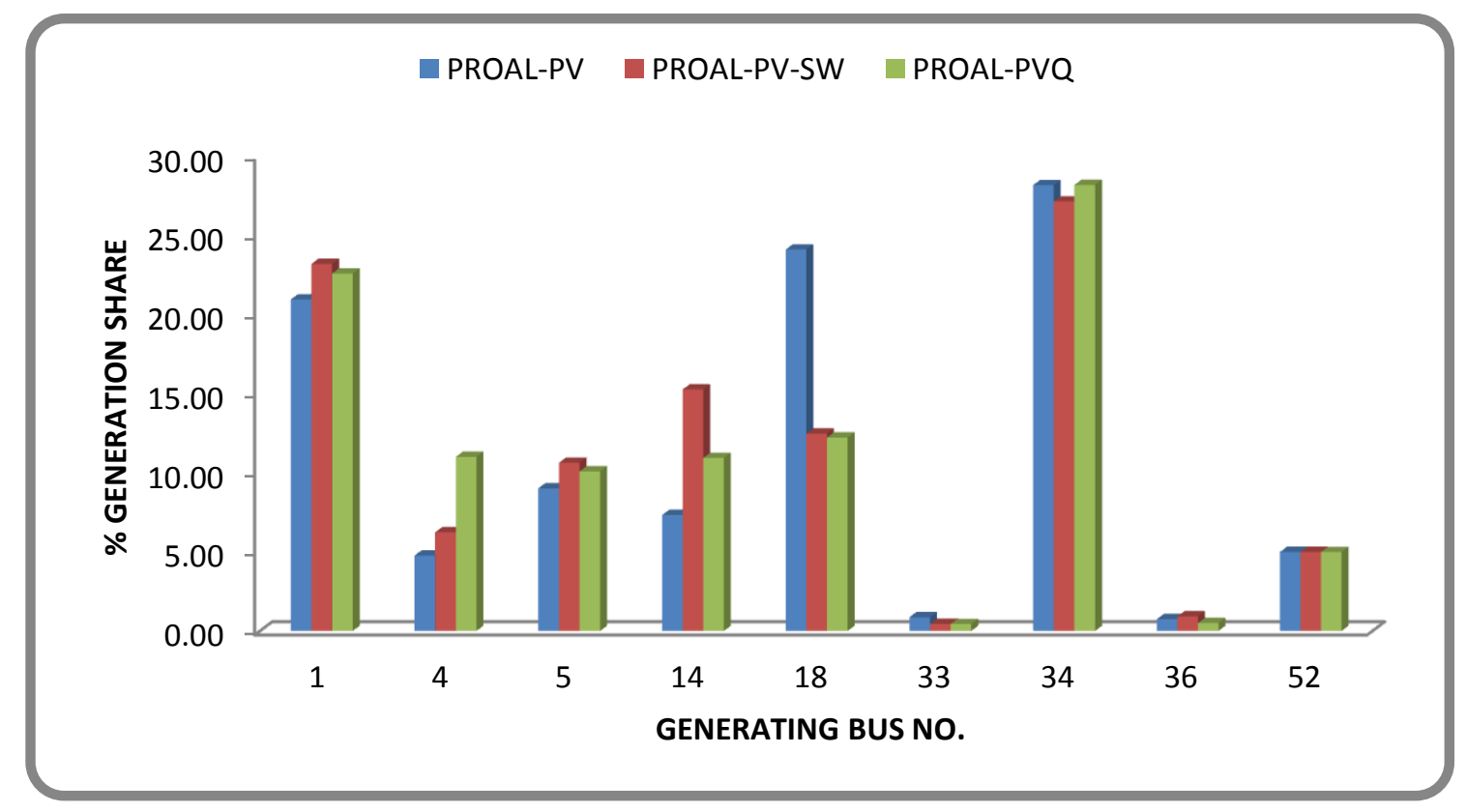

Figure (5.5): \% Real Power generation allocation during with contingency condition (Line 64) 
Electrical \& Computer Engineering: An International Journal (ECIJ) Volume 3, Number 4, December 2014

Table (5.7): \% Real Power generation allocation during with contingency condition

\begin{tabular}{|l|l|l|l|}
\hline Bus No. & PROAL-PV & PROAL-PV-SW & PROAL-PVQ \\
\hline 1 & 20.96 & 23.21 & 22.60 \\
\hline 4 & 4.77 & 6.24 & 11.00 \\
\hline 5 & 9.02 & 10.63 & 10.09 \\
\hline 14 & 7.33 & 15.27 & 10.94 \\
\hline 18 & 24.11 & 12.47 & 12.22 \\
\hline 33 & 0.87 & 0.45 & 0.44 \\
\hline 34 & 28.18 & 27.15 & 28.20 \\
\hline 36 & 0.75 & 0.92 & 0.50 \\
\hline 52 & 4.99 & 5.00 & 4.99 \\
\hline \% Total Gen. & 101.00 & 101.33 & 100.98 \\
\hline \% Total Load & 100.00 & 100.00 & 100.00 \\
\hline \% Loss & 1.00 & 1.33 & 0.98 \\
\hline
\end{tabular}

Table (5.8), shows the comparative line flows obtained using the proposed algorithms with and without contingency.

Table (5.8): Comparative transmission line flow with and without contingency

\begin{tabular}{|c|c|c|c|c|c|c|}
\hline \multirow[b]{2}{*}{$\begin{array}{l}\text { Line } \\
\text { No. }\end{array}$} & \multicolumn{3}{|c|}{ Without Contingency } & \multicolumn{3}{|c|}{ With Contingency } \\
\hline & PROAL-PV & \begin{tabular}{|l|} 
PROAL- \\
PV-SW
\end{tabular} & $\begin{array}{l}\text { PROAL- } \\
\text { PVQ }\end{array}$ & PROAL-PV & $\begin{array}{l}\text { PROAL- } \\
\text { PV-SW }\end{array}$ & $\begin{array}{l}\text { PROAL- } \\
\text { PVQ }\end{array}$ \\
\hline 1 & 0.064 & 0.061 & 0.068 & -0.071 & -0.110 & -0.013 \\
\hline 2 & 0.211 & 0.215 & 0.211 & -0.254 & -0.361 & 0.014 \\
\hline 3 & 0.044 & 0.029 & 0.048 & -0.053 & -0.077 & 0.036 \\
\hline 4 & 0.039 & 0.026 & 0.043 & -0.047 & -0.069 & 0.033 \\
\hline 5 & -0.040 & -0.032 & -0.032 & -0.005 & -0.005 & 0.023 \\
\hline 6 & -0.059 & -0.051 & -0.050 & 0.014 & 0.013 & 0.041 \\
\hline 7 & 0.023 & 0.021 & 0.020 & 0.015 & 0.013 & 0.009 \\
\hline 8 & 0.030 & 0.059 & 0.023 & -0.041 & -0.092 & 0.047 \\
\hline 9 & 0.010 & 0.040 & 0.003 & -0.016 & -0.050 & 0.028 \\
\hline 10 & 0.077 & 0.089 & 0.081 & -0.116 & -0.150 & -0.090 \\
\hline 11 & 0.072 & 0.095 & 0.080 & -0.151 & -0.217 & -0.099 \\
\hline 12 & 0.023 & 0.038 & 0.028 & -0.071 & -0.111 & -0.038 \\
\hline 13 & 0.023 & 0.038 & 0.028 & -0.071 & -0.111 & -0.038 \\
\hline 14 & 0.219 & 0.208 & 0.201 & -0.099 & -0.141 & -0.159 \\
\hline 15 & 0.038 & 0.034 & 0.034 & 0.009 & 0.007 & -0.002 \\
\hline 16 & 0.038 & 0.034 & 0.034 & -0.002 & -0.004 & -0.015 \\
\hline 17 & 0.038 & 0.034 & 0.034 & -0.003 & -0.006 & -0.017 \\
\hline 18 & 0.027 & 0.027 & 0.025 & -0.015 & -0.023 & -0.019 \\
\hline 19 & -0.033 & -0.027 & -0.028 & 0.016 & 0.013 & 0.037 \\
\hline 20 & -0.034 & -0.004 & -0.042 & 0.025 & -0.018 & 0.094 \\
\hline 21 & -0.018 & 0.010 & -0.024 & 0.007 & -0.034 & 0.070 \\
\hline 22 & 0.005 & 0.001 & 0.007 & -0.002 & 0.002 & -0.011 \\
\hline 23 & 0.059 & 0.012 & 0.076 & -0.060 & -0.014 & -0.161 \\
\hline 24 & 0.084 & 0.055 & 0.094 & -0.084 & -0.056 & -0.147 \\
\hline 25 & -0.017 & -0.013 & -0.019 & 0.021 & 0.017 & 0.030 \\
\hline 26 & 0.047 & 0.000 & 0.064 & -0.055 & -0.008 & -0.158 \\
\hline 27 & 0.019 & -0.013 & 0.031 & -0.024 & 0.009 & -0.094 \\
\hline 28 & 0.002 & 0.014 & 0.007 & -0.042 & -0.074 & -0.015 \\
\hline
\end{tabular}




\begin{tabular}{|c|c|c|c|c|c|c|}
\hline 29 & -0.010 & 0.003 & -0.005 & -0.035 & -0.070 & -0.005 \\
\hline 30 & -0.017 & 0.005 & -0.009 & -0.059 & -0.117 & -0.010 \\
\hline 31 & -0.027 & -0.027 & -0.025 & 0.034 & 0.041 & 0.040 \\
\hline 32 & 0.184 & 0.184 & 0.183 & -0.191 & -0.192 & -0.188 \\
\hline 33 & 0.112 & 0.112 & 0.112 & -0.114 & -0.114 & -0.111 \\
\hline 34 & 0.019 & 0.019 & 0.019 & -0.016 & -0.016 & -0.016 \\
\hline 35 & -0.083 & -0.083 & -0.083 & 0.080 & 0.079 & 0.079 \\
\hline 36 & 0.103 & 0.103 & 0.103 & -0.104 & -0.104 & -0.102 \\
\hline 37 & 0.103 & 0.103 & 0.103 & -0.104 & -0.104 & -0.102 \\
\hline 38 & 0.104 & 0.104 & 0.104 & -0.104 & -0.104 & -0.103 \\
\hline 39 & 0.083 & 0.083 & 0.083 & -0.082 & -0.082 & -0.082 \\
\hline 40 & 0.025 & 0.025 & 0.025 & -0.025 & -0.025 & -0.025 \\
\hline 41 & 0.045 & 0.042 & 0.050 & -0.054 & -0.092 & 0.004 \\
\hline 42 & -0.125 & -0.115 & -0.135 & 0.145 & 0.232 & 0.009 \\
\hline 43 & 0.147 & 0.147 & 0.148 & -0.174 & -0.243 & 0.002 \\
\hline 44 & 0.015 & 0.015 & 0.016 & -0.019 & -0.034 & 0.021 \\
\hline 45 & 0.049 & 0.048 & 0.050 & -0.066 & -0.112 & 0.062 \\
\hline 46 & 0.072 & 0.078 & 0.061 & -0.082 & -0.072 & 0.016 \\
\hline 47 & 0.021 & 0.017 & 0.024 & -0.032 & -0.059 & -0.051 \\
\hline 48 & 0.000 & 0.000 & 0.000 & 0.000 & 0.000 & 0.000 \\
\hline 49 & 0.000 & 0.000 & 0.000 & 0.000 & 0.000 & 0.000 \\
\hline 50 & -0.071 & -0.076 & -0.066 & 0.054 & 0.019 & 0.028 \\
\hline 51 & -0.071 & -0.076 & -0.066 & 0.054 & 0.019 & 0.028 \\
\hline 52 & 0.117 & 0.104 & 0.131 & -0.148 & -0.272 & 0.045 \\
\hline 53 & 0.126 & 0.130 & 0.122 & -0.113 & -0.086 & -0.092 \\
\hline 54 & 0.047 & 0.046 & 0.046 & -0.030 & -0.033 & -0.031 \\
\hline 55 & 0.000 & 0.000 & 0.000 & 0.007 & 0.006 & 0.007 \\
\hline 56 & -0.136 & -0.137 & -0.135 & 0.056 & 0.019 & 0.102 \\
\hline 57 & -0.136 & -0.137 & -0.135 & 0.056 & 0.019 & 0.102 \\
\hline 58 & 0.138 & 0.138 & 0.135 & -0.081 & -0.081 & -0.074 \\
\hline 59 & 0.138 & 0.138 & 0.135 & -0.081 & -0.081 & -0.074 \\
\hline 60 & 0.138 & $\begin{array}{l}0.138 \\
\end{array}$ & 0.136 & -0.081 & -0.081 & -0.074 \\
\hline 61 & 0.031 & 0.018 & 0.035 & 0.132 & 0.031 & 0.082 \\
\hline 62 & 0.000 & 0.000 & 0.000 & 0.014 & 0.012 & 0.014 \\
\hline 63 & 0.232 & 0.233 & 0.233 & -0.386 & -0.397 & -0.396 \\
\hline 64 & 0.062 & 0.062 & 0.062 & 0.000 & 0.000 & 0.000 \\
\hline 65 & 0.000 & 0.000 & 0.000 & 0.003 & 0.003 & 0.003 \\
\hline 66 & 0.000 & 0.000 & 0.000 & 0.002 & 0.002 & 0.002 \\
\hline 67 & 0.000 & 0.000 & 0.000 & 0.003 & 0.003 & 0.003 \\
\hline 68 & 0.132 & 0.132 & 0.130 & -0.077 & -0.077 & -0.071 \\
\hline 69 & 0.132 & 0.132 & 0.130 & -0.077 & -0.077 & -0.071 \\
\hline 70 & 0.000 & 0.000 & 0.000 & 0.011 & 0.011 & 0.012 \\
\hline 71 & 0.000 & 0.000 & 0.000 & 0.010 & 0.010 & 0.011 \\
\hline 72 & 0.044 & 0.044 & 0.044 & -0.042 & -0.042 & -0.042 \\
\hline 73 & 0.004 & 0.004 & 0.004 & 0.002 & 0.001 & 0.002 \\
\hline 74 & 0.000 & 0.000 & 0.000 & 0.001 & 0.000 & 0.000 \\
\hline 75 & 0.004 & 0.004 & 0.004 & 0.001 & 0.000 & 0.001 \\
\hline 76 & -0.004 & -0.004 & -0.004 & -0.001 & 0.000 & 0.000 \\
\hline 77 & 0.004 & 0.004 & 0.004 & 0.000 & 0.000 & 0.000 \\
\hline 78 & 0.004 & 0.004 & 0.004 & 0.000 & 0.000 & 0.000 \\
\hline 79 & 0.000 & 0.000 & 0.000 & 0.000 & 0.000 & 0.000 \\
\hline
\end{tabular}




\begin{tabular}{|l|l|l|l|l|l|l|}
\hline 80 & 0.000 & 0.000 & 0.000 & 0.000 & 0.000 & 0.000 \\
\hline 81 & 0.000 & 0.000 & 0.000 & 0.000 & 0.000 & 0.000 \\
\hline 82 & 0.229 & 0.229 & 0.229 & -0.355 & -0.365 & -0.365 \\
\hline 83 & 0.173 & 0.174 & 0.173 & -0.294 & -0.304 & -0.305 \\
\hline 84 & -0.149 & -0.149 & -0.148 & 0.098 & 0.089 & 0.087 \\
\hline 85 & -0.180 & -0.126 & -0.197 & 0.168 & 0.110 & 0.287 \\
\hline 86 & -0.180 & -0.126 & -0.197 & 0.168 & 0.110 & 0.287 \\
\hline 87 & -0.049 & -0.048 & -0.050 & 0.066 & 0.112 & -0.062 \\
\hline 88 & -0.133 & -0.132 & -0.134 & 0.153 & 0.215 & -0.016 \\
\hline
\end{tabular}

\subsection{SGA based OPF using Fuzzy Penalty}

The fuzzy based penalty is being implemented for further improvement of the convergence criterion of SGA based proposed algorithms on the $220 \mathrm{kV}$ systems. The statistical parameters obtained after 10 runs of PROAL-PVQ for sample probability ordered pair $(0.1,0.1)$ and $(0.7$, 0.5 ) are shown in Table (5.9). From table (5.10), it is observed that Algorithms function value improves with fuzzy based penalty at the cost of slight increase in computational time. The same conclusions can be drawn for with contingency condition from table (5.11) and (5.12).

F-Penalty with fixed penalty; FP- Penalty with Fuzzy penalty

Table (5.9): Comparison of fuel cost for fixed and fuzzy penalty for PROAL-PVQ (without contingency)

\begin{tabular}{|l|l|l|l|l|}
\hline \multirow{2}{*}{ Parameter } & \multicolumn{2}{l|}{$\mathrm{CV}=0.1, \mu=0.1$} & \multicolumn{2}{l|}{$\mathrm{CV}=0.7, \mu=0.5$} \\
\cline { 2 - 5 } & $\mathrm{F}$ & $\mathrm{FP}$ & $\mathrm{F}$ & $\mathrm{FP}$ \\
\hline Average & 8.96 & 8.19 & 8.95 & 8.27 \\
\hline Minimum & 8.86 & 8.17 & 8.91 & 8.23 \\
\hline Maximum & 9.59 & 8.23 & 8.96 & 8.34 \\
\hline Kurtosis & 7.88 & 2.62 & 3.47 & 2.86 \\
\hline Skewness & 2.59 & 0.919 & -1.22 & 1.12 \\
\hline Std. deviation & 0.22 & 0.019215 & 0.016 & 0.038 \\
\hline
\end{tabular}

Table (5.10): Comparison of computational time for fixed and fuzzy penalty for PROAL-PVQ (without contingency)

\begin{tabular}{|l|l|l|l|l|}
\hline \multirow{2}{*}{ Parameter } & \multicolumn{2}{|l|}{$\mathrm{CV}=0.1, \mu=0.1$} & \multicolumn{2}{l|}{$\mathrm{CV}=0.7, \mu=0.5$} \\
\cline { 2 - 5 } & $\mathrm{F}$ & $\mathrm{FP}$ & $\mathrm{F}$ & $\mathrm{FP}$ \\
\hline Average & 69.44 & 74.49 & 59.93 & 60.75 \\
\hline Minimum & 39.57 & 56.09 & 54.01 & 53.35 \\
\hline Maximum & 87.21 & 99.17 & 64.35 & 66.875 \\
\hline
\end{tabular}

Table (5.11): Fuel cost Comparison for fixed and fuzzy penalty for PROAL-PVQ (with contingency)

\begin{tabular}{|l|l|l|l|l|}
\hline \multirow{2}{*}{ Parameter } & \multicolumn{2}{|l|}{$\mathrm{CV}=0.1, \mu=0.1$} & \multicolumn{2}{l|}{$\mathrm{CV}=0.7, \mu=0.5$} \\
\cline { 2 - 5 } & $\mathrm{F}$ & $\mathrm{FP}$ & $\mathrm{F}$ & $\mathrm{FP}$ \\
\hline Average & 10.67 & 8.26 & 10.67 & 8.33 \\
\hline Minimum & 10.53 & 8.23 & 10.62 & 8.25 \\
\hline Maximum & 11.42 & 8.41 & 10.70 & 8.4654 \\
\hline Kurtosis & 7.94 & 7.32 & 1.59 & 2.65 \\
\hline Skewness & 2.61 & 2.41 & -0.67 & 0.87 \\
\hline Std. deviation & 0.26 & 0.0520 & 0.039 & 0.075 \\
\hline
\end{tabular}


Table (5.12): Comparison of computational time for fixed and fuzzy penalty for PROAL-PVQ (with contingency)

\begin{tabular}{|l|l|l|l|l|}
\hline \multirow{2}{*}{ Parameter } & \multicolumn{2}{|l|}{$\mathrm{CV}=0.1, \mu=0.1$} & \multicolumn{2}{l|}{$\mathrm{CV}=0.7, \mu=0.5$} \\
\cline { 2 - 5 } & $\mathrm{F}$ & $\mathrm{FP}$ & $\mathrm{F}$ & $\mathrm{FP}$ \\
\hline Average & 59.92 & 67.17 & 65.81 & 64.02 \\
\hline Minimum & 48.125 & 51.79 & 53.125 & 50.76 \\
\hline Maximum & 75.31 & 88.625 & 87.54 & 88.56 \\
\hline
\end{tabular}

\subsection{Congestion management}

The proposed GA based algorithms are also tested for obtaining available transfer capability of system under various contingency conditions. ATC study gives the idea of additional amount of power above some base case value that can be transferred in a prescribed manner between two sets of buses where the source, in which power injections are increased, and the sink, in which power injections are decreased by an offsetting amount. The operational or physical limit to be reached that prevents further increase in the transfer power and the loading in the network. The effects of contingencies are taken into account in the determination.

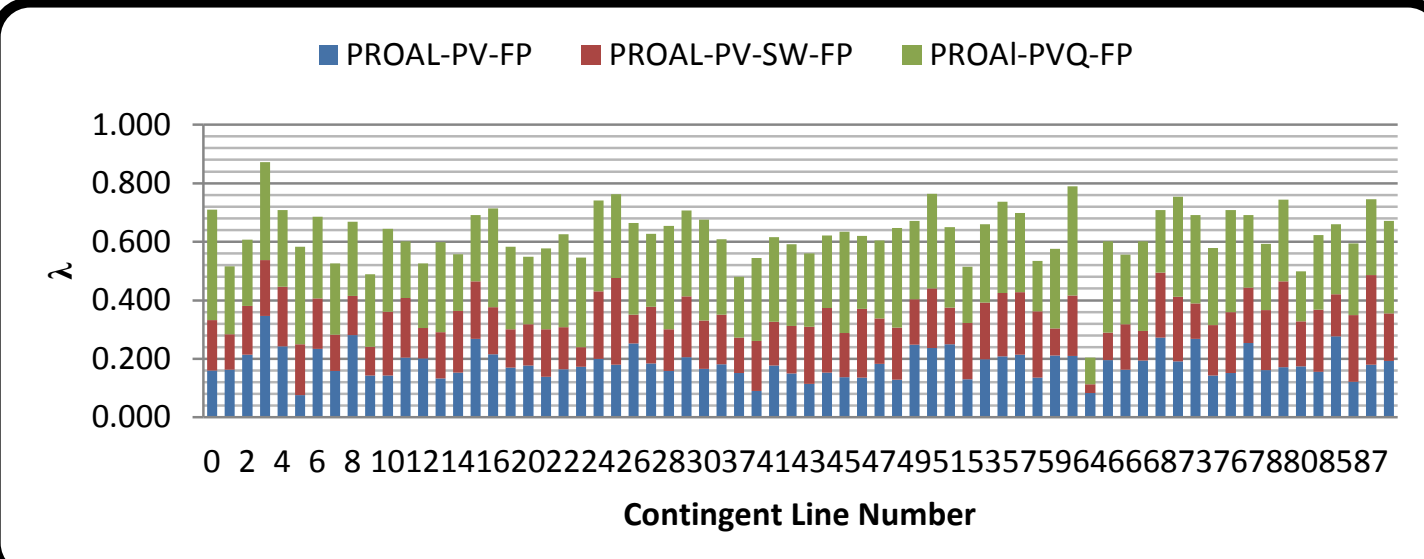

Figure (5.6): The comparative maximum load transfer $\lambda$ calculated using proposed fuzzy penalty based GA OPF algorithms

Formulation of the ATC problem is in terms of a single unknown, the scalar parameter $\lambda$, whose value is to be maximized for each ATC case. The available transfer capacity is determined for Single contingency case is processed by GA based proposed algorithms to find the maximum transfer power without causing a limit violation. The proposed fuzzy penalty based proposed algorithms PROAL-PV-FP, PROAL-PV-SW-FP and PROAL-PVQ-FP are used to calculate, the maximum load transfer on the system. For every method, 10 runs of algorithms are carried out and averages of various are calculated for conclusion purpose.

The maximum load transfer capability calculated using various proposed algorithms PROALPV-FP; PROAL-PV-SW-FP and PROAL-PVQ-FP are shown in figure (5.6) and also given in table (5.13).

Table (5.13): The maximum load transfer $\lambda$ obtained through proposed algorithms 


\begin{tabular}{|c|c|c|c|c|c|c|c|}
\hline \multirow[b]{2}{*}{ TLC } & \multicolumn{3}{|c|}{$\lambda$} & \multirow[b]{2}{*}{ TLC } & \multicolumn{3}{|c|}{$\lambda$} \\
\hline & $\begin{array}{l}\text { PROAL- } \\
\text { PV-FP }\end{array}$ & $\begin{array}{l}\text { PROAL-PV- } \\
\text { SW-FP }\end{array}$ & $\begin{array}{l}\text { PROAL- } \\
\text { PVQ-FP }\end{array}$ & & $\begin{array}{l}\text { PROAL-PV- } \\
\text { FP }\end{array}$ & $\begin{array}{l}\text { PROAL-PV- } \\
\text { SW-FP }\end{array}$ & $\begin{array}{l}\text { PROAL- } \\
\text { PVQ-FP }\end{array}$ \\
\hline 0 & 0.160 & 0.173 & 0.378 & 43 & 0.115 & 0.195 & 0.250 \\
\hline 1 & 0.163 & 0.122 & 0.231 & 44 & 0.152 & 0.221 & 0.249 \\
\hline 2 & 0.214 & 0.166 & 0.227 & 45 & 0.137 & 0.152 & 0.346 \\
\hline 3 & 0.346 & 0.191 & 0.336 & 46 & 0.136 & 0.235 & 0.249 \\
\hline 4 & 0.243 & 0.204 & 0.261 & 47 & 0.182 & 0.156 & 0.266 \\
\hline 5 & 0.076 & 0.174 & 0.333 & 48 & 0.128 & 0.179 & 0.339 \\
\hline 6 & 0.235 & 0.172 & 0.278 & 49 & 0.249 & 0.155 & 0.267 \\
\hline 7 & 0.158 & 0.125 & 0.243 & 50 & 0.237 & 0.204 & 0.324 \\
\hline 8 & 0.282 & 0.134 & 0.254 & 51 & 0.250 & 0.125 & 0.275 \\
\hline 9 & 0.143 & 0.097 & 0.249 & 52 & 0.131 & 0.192 & 0.192 \\
\hline 10 & 0.144 & 0.217 & 0.284 & 53 & 0.198 & 0.193 & 0.269 \\
\hline 11 & 0.204 & 0.205 & 0.193 & 56 & 0.209 & 0.215 & 0.313 \\
\hline 12 & 0.202 & 0.104 & 0.220 & 57 & 0.214 & 0.214 & 0.270 \\
\hline 13 & 0.133 & 0.158 & 0.309 & 58 & 0.135 & 0.227 & 0.173 \\
\hline 14 & 0.153 & 0.210 & 0.194 & 59 & 0.212 & 0.092 & 0.272 \\
\hline 15 & 0.269 & 0.195 & 0.227 & 60 & 0.210 & 0.207 & 0.373 \\
\hline 16 & 0.216 & 0.161 & 0.336 & 64 & 0.083 & 0.031 & 0.091 \\
\hline 19 & 0.170 & 0.131 & 0.282 & 65 & 0.196 & 0.093 & 0.313 \\
\hline 20 & 0.177 & 0.142 & 0.230 & 66 & 0.162 & 0.155 & 0.238 \\
\hline 21 & 0.138 & 0.162 & 0.277 & 67 & 0.194 & 0.102 & 0.306 \\
\hline 22 & 0.165 & 0.143 & 0.318 & 68 & 0.272 & 0.223 & 0.213 \\
\hline 23 & 0.173 & 0.066 & 0.306 & 69 & 0.191 & 0.222 & 0.341 \\
\hline 24 & 0.200 & 0.231 & 0.311 & 73 & 0.269 & 0.120 & 0.302 \\
\hline 25 & 0.180 & 0.297 & 0.286 & 75 & 0.144 & 0.172 & 0.264 \\
\hline 26 & 0.252 & 0.098 & 0.314 & 76 & 0.152 & 0.208 & 0.349 \\
\hline 27 & 0.184 & 0.194 & 0.249 & 77 & 0.254 & 0.189 & 0.249 \\
\hline 28 & 0.158 & 0.143 & 0.353 & 78 & 0.162 & 0.204 & 0.227 \\
\hline 29 & 0.205 & 0.209 & 0.292 & 79 & 0.172 & 0.293 & 0.278 \\
\hline 30 & 0.165 & 0.166 & 0.344 & 80 & 0.174 & 0.154 & 0.170 \\
\hline 36 & 0.181 & 0.170 & 0.258 & 81 & 0.155 & 0.213 & 0.254 \\
\hline 37 & 0.152 & 0.120 & 0.207 & 85 & 0.277 & 0.144 & 0.239 \\
\hline 39 & 0.091 & 0.171 & 0.283 & 86 & 0.122 & 0.228 & 0.244 \\
\hline 41 & 0.177 & 0.150 & 0.288 & 87 & 0.180 & 0.306 & 0.259 \\
\hline 42 & 0.150 & 0.163 & 0.279 & 88 & 0.193 & 0.163 & 0.316 \\
\hline
\end{tabular}

Figure (5.6) and Table (5.13) shows that the proposed method PROAL-PVQ with fuzzy penalty provides better estimate of $\lambda$ amongst all other methods. Referring to this table, in $83 \%$ contingency conditions, this algorithm has provided the increased load transfer capability for system. The table (5.13) shows maximum value of $\lambda$ out 10 runs conducted for each contingency condition for ordered pair of $(0.85,0.1)$.

\section{CONCLUSION}

Among all the methods presented the method PROAL-PVQ produces the better results in terms of cost of generation, computation time, system conditions and Transmission line flows with fixed penalty. Also the congestion management provided by this method is better than provided by other methods. 


\section{REFERENCES:}

[1] M. M. El-Saadawi, "A Genetic-Based Approach for Solving Optimal Power Flow Problem", Mansoura Engineering Journal, (MEJ), Vol. 29, No. 2, June 2004

[2] J. Yuryevich, K. P. Wong, "Evolutionary Programming Based Optimal Power Flow Algorithm", IEEE Transaction on Power Systems, Vol. 14, No. 4, November 1999

[3] T. Numnonda and U. D. Annakkage, "Optimal power dispatch in multinode electricity market using genetic algorithm,” Electric Power System Research., vol. 49, 1999, pp. 211-220.

[4] A Genetic Algorithm for Solving the Optimal Power Flow Problem Tarek BOUKTIR, Linda SLIMANI, M. BELKACEMI,

[5] Mithun M. Bhaskar, Sydulu Maheswarapu, “A Hybrid Genetic Algorithm Approach for Optimal Power Flow", TELKOMNIKA, Vol.9, No.2, August 2011, pp. 211 216

[6] C.M.Wankhade, A.P.Vaidya,"Comparative Study of GA Based Optimal Power Flow", International Journal on Advance Electrical and Electronics Engineering, on 28 June 12, Vol.1, Issue-1, 20-25.

[7] C.M.Wankhade,B.P.Saoji,A.P.Vaidya,’Optimal Power Flow Evaluation of Power System using Genetic Algorithm "International Journal of Power System Operation and Energy Management, Dec 2012 ,Vol.1,Issue-4, 67-72.

[8] C.M.Wankhade, A.P.Vaidya,’Optimal Power Flow using Genetic Algorithm: Parametric Studies for selection of Control \& States Variables", British Journal of Applied Science \& Technology, published on Feb 2014, Vol.4, Issue-2,279-301.

[9] C.M.Wankhade, A.P.Vaidya,"'OPTIMAL POWER FLOW BASED CONVENTIONAL METHODS FOR CONGESTION MANAGEMENT” IASTER journal, published on Jan-March 2014, Vol.2, Issue-1,01-11.

[10] C.M.Wankhade, A.P.Vaidya," Application of GA to decide the available Load Transfer Capability of the System" IJAREEIE Journal, published on Jan 2014, Vol.3, Issue-1,01-09. 